International Journal on Intelligent Electronic Systems, Vol. 6 No. 2 July 2012

\title{
A REVIEW ON OBSTACLE AVOIDANCE AND PATH PLANNING OF ROBOTIC SYSTEMS IN CLUTTERED ENVIRONMENTS
}

\author{
Nazemizadeh M. \\ Young Researchers Club, Damavand Branch, Islamc Azad University, Damavand, Iran. \\ Email:mn.nazemizadeh@gmail.com
}

\begin{abstract}
This paper presents a review on some conventional method of path planning of robotic systems in cluttered environment. Hence, the complexity and uncertainty of the path planning are increased in presence of environmental obstacles, this matter has been received a great deal of interest, recently. In this work, some conventional method on the obstacle avoidance of the robots such as potential field method, road map method and cell decomposition method are presented, and the effectiveness and defects of these methods are discussed. Moreover, some advanced methods on the obstacle avoidance and the path planning of the fixed and mobile robots are reviewed and discussed including Genetic Algorithm, Neural Network, Fuzzy logic, Ant Colony, Particle Swarm Optimization, Wavelet Theory, Tabu Search.
\end{abstract}

Key words: Robotic systems, Obstacle avoidance, Path planning, Potential field method, Road map method, Cell decomposition.

\section{INTRODUCTION}

Robot motion can generally categorized into three steps: at first, the robot must be able to determine its position and configuration in its workspace, this level is known as Localization, and constructs a distinct area of researches in the robotic field. Next step is known as path planning, in which the robot must plan its motion from a given start configuration to a goal position. Finally, the robot must be able to control its desired path during the motion, which is named as motion control. Hence any of these three areas include many developed methods and procedures, amount of research papers have been only focused on the path planning of the systems. Figure (1) shows this classification. Beside, the robotic systems operate in a wide range of environments, including variant type of obstacles. Thus, the system must be able to plan its motion without colliding to obstacles. To plan the point-to-point motion of the robots in the cluttered environments, a great deal of methods have been developed, which in this paper is presented and discussed. The path planning in the presence of the obstacles are classified as global and local path planning [1]. In the local techniques, the environmental map is not completely known and the trajectory planning of the mobile robot is mainly based on its variant sensors information [2]. However, since the accuracy of sensors is vital in the motion planning problems, the use of global techniques has been recognized as a possible solution to planning the mobile robotic system trajectories. In the global techniques, the robot's environment is completely known and the trajectory planning is defined as the modeling of the obstacles. Hence, problem algorithm is identifying which makes the mobile robot move in the environment without colliding to the obstacles. The global approaches are basically categorized as road map (RM) method, cell decomposition (CD) algorithm and potential field (PF) method. Moreover, some advanced approaches are treated in this paper.

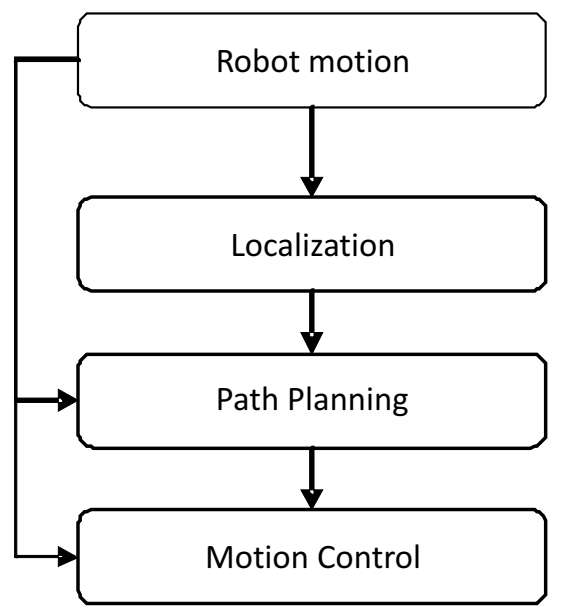

Fig. 1 Robot motion steps

\section{ROAD MAP METHOD}

In this method, the continuous environment is modeled into a set of routes within the free space. Then, these set of roads (including straight lines and curves) construct a network of paths, named road map. On the other word, the road map is a network of short 
segments which totally set up a road network [3]. Thus, the free space is converted to a set of admissible roads. Beside, the challenge is to construct these set of roads that together enable the robot to go anywhere in its free space. The approaches of modeling the free space to a set of can be treated as different approaches. In the case of the Visibility graph, roads come as close as possible to obstacles and resulting paths are minimum-length solutions. In the case of the Voronoi diagram, roads stay as far away as possible from obstacles [1].

The Visibility graph (VG) is constructed by a collection of lines in the free space that connects an edge of an obstacle to that of another. It means that two vertices are connected in the graph if they are mutually visible [4]. Asano et al. [5] used the Visibility approach to set a road map among polygon obstacles, and then presented a shortest path between start and goal points. Moreover, an efficient algorithm for planning collision-free motion of a convex polygonal object in 2-dimensional space amidst polygonal obstacles is presented in $[6,7]$ based on VG. Rekleitis et al. [8] used a VG- like procedure for path planning of multiple robots. Moreover, an enhanced version of this method is developed in [9] to collision-free path planning of mobile robots in dynamic environment.

Another approach is known as Voronoi diagram (VD). In despite of VG approach, in VD method, the road map construction tends to maximize the distance between the robot and the obstacles in the map [10]. The Voronoi diagram consists of the lines constructed from all points that are equidistant from two or more obstacles. Canny [11] proposed VD approach for path planning in cluttered environments. Schwartz and Sharir [12] studied a VD approach to plan the path of an object amidst polygon barriers. Moreover, an algorithm to move a disc among polygons using the Voronoi diagram is reported in [13]. Amato and Wu [14] employed an advanced VD method for path and manipulation planning of robotic systems. Furthermore, an optimal path planner with obstacle avoidance strategy is developed in [15]. Dong et al. [16] proposed an advanced path planning method for mobile manipulators based on VD, and Breitenmoser et al. [17] studied convergence of VD method for non-convex environments with a group of networked robots.

As the presented researches are based on a graph model of Network of roads using vertices of obstacles, the backbone of these models is dependent upon the nature and clutter of the environment. Thus, model environment mapping methods for a convex environment offer elegant solutions, but these methods require accurate sensor information and are therefore difficult to implement in practice.

\section{CELL DECOMPOSITION METHOD}

In fact, the main idea of the cell decomposition method (CDM) is to discriminate between geometric areas, or cells, that are free and areas that are occupied by obstacles [18]. The basic cell decomposition path-planning algorithm can be summarized as: dividing the workspace into a set of free and occupied cells or grids, connecting adjacent free cells and constructing a graph named connectivity graph, and plan the shortest path among the paths of connectivity graph. In the literature review, an important aspect of cell decomposition methods is known as the placement of the boundaries between cells [1]. If the boundaries are placed as a function of the structure of the environment, such that the decomposition is lossless, then the method is termed exact cell decomposition. If the decomposition results in an approximation of the actual map, the system is termed approximate cell decomposition. Keil and Sack [19] decomposed the environment via the vertices of polygon obstacles which is assumed as the exact cell decomposition. Brooks [20] developed a three dimensional global path planner for a PUMA robot arm based on $C D$ method. Furthermore, a procedure to fill the whole region with obstacle avoidance for a robot using a raster scanning strategy is developed in [21], which is related to the sensor based decomposition. Fujimura and Samet [22] presented a hierarchical strategy for path planning among moving obstacles based on CD algorithm. Choset [23] proposed a novel cellular decomposition approach by breaking down the workspace. This solution combines the advantages of cell decomposition and template based approaches, and minimizes the number of cells used in cell decomposition. Moreover, an improved CD method is presented in [24], which tends to ensure the convergence of method. Atkar et al. [25] applied the exact cellular decomposition to complete coverage of a closed orientable surface in three dimensions. Chenghui and Ferrari [26] developed a novel approximate cell-decomposition method in which obstacles, targets, and sensor's platform are represented as closed and bounded subsets of an 
Euclidean workspace. Their method constructed a connectivity graph with observation cells that is pruned and transformed into a decision tree from which an optimal sensing strategy can be computed.

Moreover, a simple feedback law is proposed over a given cell decomposition of obstructed environments is presented in [27]. Swingler and Ferrari [28] proposed a cell decomposition approach to cooperative path planning and collision avoidance. Furthermore, a path planning method for palletizing tasks of an industrial 6-axis robot using workspace grid decomposition is presented in [29].

Hence the cell decomposition algorithms explicitly computes the configuration space of the mobile robot, decomposes the resulting space into cells, and then searches for a route in the free space cell graph. However, the algorithm suffers from the drawback of high time complexity [30].

\section{POTENTIAL FIELD METHOD}

Potential field (PF) method is a novel method, which has a elegant mathematics and simplicity. In this method, path planning methodology creates a field, or gradient, across the robot's map that directs the robot to the goal position from multiple prior positions [31]. The basic concept of potential field method is to fill the robot's workspace with an artificial potential field, in where the robot is repulsed away from the obstacles and is attracted to its goal position. In this method, if the coordinate of the robot is considered as, and the artificial potential field is considered as, this filed is given as:

$$
U(q)=U_{\text {att }}(q)+U_{\text {rep }}(q)
$$

Where $U_{\text {att }}$ is the attractive field of the goal, and $U_{\text {rep }}$ is the repulsive field of the obstacles.

Then, the gradient of this artificial potential is assumed as a force vector which is applied to the dynamic of the system [31]:

$$
F(q)=-\nabla U(q)
$$

The first formulation of potential field method has been addressed to Khatib [31]. He presented the PF method for the path planning of the mobile robots and manipulators in an obstructed environment. His proposed potential field formulation is given as [32]:

$$
\begin{aligned}
& U_{\text {att }}(q)=\frac{1}{2} k_{\text {att }} \rho_{\text {goal }}^{2}(q)
\end{aligned}
$$

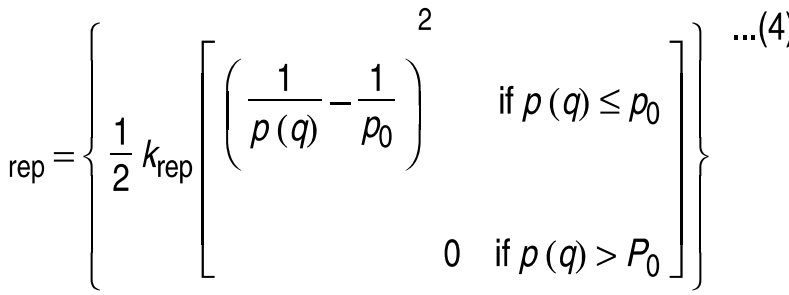

Newman and Hogan [33] used dynamic potential functions to control of high speed robot motion. Borenstein and Koren [34] proposed an obstacle avoidance procedure for fact mobile robots based on PF method. Moreover, they [35] discussed the inherent limitations such as local minima, multiple obstacles. Thus, a lot of procedures and potential functions are proposed to rectify these limitations [36, 37]. Kim and Khosla [38] proposed a harmonic potential function to avoid the limitations of PF method. Moreover, a curvature-velocity method for local obstacle avoidance is presented in [39]. Ge [40] proposed a new potential function to collision avoidance of the mobile manipulators. Park et al. [41] employed the PF and the simulated annealing method for obstacle avoidance of mobile robots. An analytical algorithm for the collision-free motion planning of the wheeled mobile robots is presented in [42] based on potential field method. In this work, only kinematic model of the mobile robot is considered which is simple and straight forward. However, since inertia and torque constraints are neglected in this study. Shimoda et al. [43] used the PF based navigation for high speed unmanned ground vehicles on uneven terrain. Moreover, a study of a new method for improving artificial potential field in mobile robot obstacle avoidance is presented in [44]. Huang [45] studied a velocity planning for a mobile robot to track a moving target. In this paper, the potential field method is applied for both path and speed planning or the velocity planning, for a mobile robot in a dynamic environment where the target and the obstacles are moving. The robot's planned velocity is determined by relative velocities as well as relative positions among robot, obstacles and targets. Further more, Korayem and Nazemizadeh [46] presented an optimal motion planning of the non-holonomic mobile robots in the presence of multi obstacles based on PF method. They applied the potential function to the index performance of the optimal problem, to avoid the PF limitations, and then used an indirect method to solve 
the problem. Also, Korayem and Nazemizadeh [47] developed a new formulation of potential functions using enclosed optimal ellipsoids to model the mobile manipulator parts and the environmental obstacles.

\section{ADVANCED METHODS}

In order to improve the efficiency of Classic methods, Probabilistic algorithms have been developed, including Probabilistic Roadmaps and rapidly exploring Random Trees [48-51], with major advantages is high-speed implementation. Also, there are many Heuristic and Mete-heuristic algorithms for mobile robot path planning such as Genetic Algorithm [52, 53], Artificial Neural Network [54, 55], Fuzzy Logic [56, 57], Ant Colony [58, 59], Particle Swarm Optimization [60, 61], Wavelet Theory [62], Tabu Search [63, 64].

\section{CONCLUSION}

This paper has presented a review on some conventional method of path planning of robotic systems in cluttered environment such as potential field method, road map method, and cell decomposition method. Also, the effectiveness and defects of these methods have been discussed. Moreover, some advanced methods for the obstacle avoidance and the path planning of the fixed and mobile robots are reviewed and discussed including Genetic Algorithm, Neural Network, Fuzzy logic, Ant Colony, Particle Swarm Optimization, Wavelet Theory, Tabu Search.

\section{REFERENCES}

[1] R. Siegwart, I. Nourbakhsh, Introduction to Autonomous Mobile Robots (MIT Press, 2004).

[2] Ch. Shi, Y. Wang, J. Young, A Local Obstacle Avoidance Method for Mobile Robots in Partially Known Environment, Robotics and Autonomous Systems, Vol. 58, pp. 425-434, 2010.

[3] J. Latombe, Robot Motion Planning (Kluwer Academic Publishers, 1991).

[4] M. Saska, M. Macas, L. Preucil, L. Lhotska, Robot Path Planning using Particle Swarm Optimization of Ferguson Splines, Proceedings of the IEEE Conference on Emerging Technologies and Factory Automation, pp. 833-839, 2006.

[5] T, Asano, L. Guibas, J. Hershberger, H. Imai, Visibidilty-polygon Search and Euclidean Shortest Path, Symposium on Foundations of Computer Science, pp. 155-164, 1985.

[6] K. Kedem, M. Sharir, An Efficient Algorithm for Planning Collision-free Motion of a Convex Polygonal
Object in 2-dimensional Space amidst Polygonal Obstacles, Proceedings of the 1st Annual ACM Symposium on Computational Geometry, pp. 75-80, 1985.

[7] K. Kedem, M. Sharir, An Automatic Motion Planning System for a Convex Polygonal Mobile Robot in 2-D Polygonal Space, Proceedings of the 4th Annual ACM Symposium on Computational Geometry, pp. 329-340, 1988.

[8] I. Rekleitis, G. Dudek, E. Milios, Multi-robot Exploration of an Unknown Environment, Efficiently Reducing the Odometry Error, International Joint Conference in Artificial Intelligence, Vol. 2, pp. 1340- 1345, 1997.

[9] P. Raja, S. Pugazhenthi, Path Planning for a Mobile Robot in Dynamic Environments, International Journal of the Physical Sciences, Vol. 6, pp. 4721-4731, 2011.

[10] J. F. Canny, The Complexity of Robot Motion Planning (MIT Press, 1987).

[11] J. F. Canny, B. Donald, Simplified Voronoi Diagram, Proceedings of ACM Symposium on Computational Geometry, pp. 153-161, 1987.

[12] J.T. Schwartz, M. Sharir, On the Piano Movers' Problem: III. Coordinating the Motion of Several Independent Bodies amidst Polygon Barriers, International Journal of Robotics Research, Vol. 2, pp. 46-75, 1983.

[13] C.O'Dunlaing, C. K. Yap, A Retraction Method for Planning the Motion of a Disc, Journal of Algorithm, Vol. 6, pp. 104-111, 1985.

[14] N.M. Amato, Y. Wu, A Randomized Roadmap Method for Path and Manipulation Planning, Proceedings of the International Conference on Robotics and Automation, pp.113-120, 1996.

[15] D.M. Coleman, J.T. Wunderlich, O3: An Optimal and Opportunistic Path Planner (with Obstacle Avoidance) Using Voronoi Polygons, Advanced Motion Control, Vol. 1, pp. 371-376, 2008.

[16] H. Dong, W. Li, J. Zhu, S. Duan, The Path Planning for Mobile Robot Based on Voronoi Diagram, Intelligent Networks and Intelligent Systems, pp. 446-449, 2010.

[17] A. Breitenmoser, M. Schwager, J. C. Metzger, R Siegwart, D. Rus, Voronoi Coverage of Non-convex Environments with a Group of Networked Robots, International Conference on Robotics and Automation, pp. 4982-4989, 2010.

[18] S. Russell, P. Norvig, Artificial Intelligence, a Modern Approach (Prentice Hall International, 1995).

[19] J.M. Keil, J. R. Sack, Minimum Decomposition of Polygonal Objects, Computational Geometry, pp. 197-216, 1985. 
[20] R.A. Brooks, Solving the Find Path Problem by Good Representation of Free Space, IEEE Transaction on Systems, Man, and Cybernetics, Vol. 3, pp. 190-197, 1985.

[21] L. Cao, Y. Y. Huang, E. L. Hall, Region Filling Operations with Random Obstacle Avoidance for Mobile Robots, Journal of Robotic Systems, Vol. 5, pp. 87-102, 1988.

[22] K. Fujimura, H. Samet, A Hierarchical Strategy for Path Planning among Moving Obstacles, Robotics and Automation, Vol.5, pp. 61-69, 1989.

[23] H. Choset, Coverage of Known Spaces: the Boustrophedon Cellular Decomposition, Autonomous Robots, Vol. 9, pp. 247-253, 2000.

[24] U. Acar, H. Choset, Critical Point Sensing in Unknown Environments, Proc. Int. Conf. Robotics Automation, pp. 3803-3810, 2000.

[25] N. Atkar, H. Choset, A. A. Rizzi, E. U. Acar, Exact Cellular Decomposition of Closed Orientable Surfaces Embedded in R3, Proc. Int. Conf. Robotics Automation, pp. 699-704, 2001.

[26] Ch, Cai, S. Ferrari, Information-Driven Sensor Path Planning by Approximate Cell Decomposition, Systems, Man, and Cybernetics, Vol. 39, pp. 672-689, 2009.

[27] S.R. Lindemann, S. M. LaValle, Simple and Efficient Algorithms for Computing Smooth, Collision-free Feedback Laws Over Given Cell Decompositions, International Journal of Robotics Research, Vol. 28, pp. 600-621, 2009.

[28] A. Swingler, S. Ferrari, A Cell Decomposition Approach to Cooperative Path Planning and Collision Avoidance via Disjunctive Programming, Conference on Decision and Control, pp. 6329-6336, 2010.

[29] C. Scheurer, U. E. Zimmermann, Path Planning Method for Palletizing Tasks Using Workspace Cell Decomposition, Conference on Robotics and Automation, pp. 1-4, 2011.

[30] R. Arkin, R. Murphy, Autonomous Navigation in a Manufacturing Environment. Robots Autonomous, Vol. 6, pp. 445-454, 1990.

[31] 0. Khatib, Real-Time Obstacle Avoidance for Manipulators and Mobile Robots, Proceedings of International Conference on Robotics and Automation, pp. 500-505, 1985.

[32] O. Khatib, Real-Time Obstacle Avoidance for Manipulators and Mobile Robots, International Journal of Robotics Research, Vol. 5, pp. 90-98, 1986.

[33] W. Newman, N. Hogan, High Speed Robot Control and Obstacle Avoidance Using Dynamic Potential Functions, International Conference on Robotics and Automation, Vol. 4, pp. 14- 24, 1987.
[34] J. Borenstein, Y. Koren, Real-time Obstacle Avoidance for Fact Mobile Robots, Transactions on Systems, Man and Cybernetics, Vol. 19, pp. 1179-1187, 1989.

[35] Y. Koren, J. Borenstein, Potential Field Methods and their Inherent Limitations for Mobile Robot Navigation, International Conference on Robotics and Automation, pp. 1398-1404, 1991.

[36] X. Yun, K. Tan, Wall-following Method for Escaping Local Minima in Potential Field Based Motion Planning, Proceeding of the 8th International Conference on Advanced Robotics, pp. 421-426, 1997.

[37] C. Sheu, K. Young, Heuristic Approach to Robot Path Planning Based on Task Requirements Using a Genetic Algorithm, Journal of Intelligent and Robotic Systems, Theory and Applications, Vol. 1, pp. 65-88, 1998.

[38] J. Kim, P. K. Khosla, Real-time Obstacle Avoidance Using Harmonic Potential Functions, Transactions on Robotics and Automation, Vol. 3, pp. 338-349, 1992.

[39] R. Simmons, The Curvature-velocity Method for Local Obstacle Avoidance, Robotics and Automation, Vol. 4, pp. 22-28, 1996.

[40] S.S. Ge, Y.J. Cui, New Potential Functions for Mobile Robot Path Planning, Robotics and Automation, vol. 16, pp. 615-620, 2000.

[41] M.G. Park, J.H. Jeon, M.Ch. Lee, Obstacle Avoidance for Mobile Robots Using Artificial Potential Field Approach with Simulated Annealing, International Symposium on Industrial Electronics, Vol. 3, pp. 1530-1535, 2001.

[42] V.J. Gonzalez, R. Parkin, M. Lopez-Para, J. M. Dorador, A Wheeled Mobile Robot with Obstacle Avoidance Capability, Mechanica Technologia, Vol. 1, pp. 150-159, 2004.

[43] S. Shimoda, Y. Kuroda, K. lagnemma, Potential Field Navigation of High Speed Unmanned Ground Vehicles on Uneven Terrain, International Conference on Robotics and Automation, pp. 2828- 2833, 2005.

[44] E. Shi, T. Cai, Ch. He, J. Guo, Study of the New Method for Improving Artificial Potential Field in Mobile Robot Obstacle Avoidance, International Conference on Automation and Logistics, pp. 282-286, 2007.

[45] L. Huang, Velocity Planning for a Mobile Robot to Track a Moving Target - a Potential Field Approach, Robotics and Autonomous Systems, Vol. 57, pp. 55-63, 2009.

[46] M.H. Korayem, M. Nazemizadeh, H. Binabaji, V. Azimirad, Optimal Motion Planning of Non-holonomic Mobile Robots in Presence of Multi Obstacles, International Conference on Emerging Trends in 
Robotics and Communication Technologies, pp. 269-272, 2010.

[47] M.H. Korayem, M. Nazemizadeh, V. Azimirad, Optimal Trajectory Planning of Wheeled Mobile Manipulators in Cluttered Environments Using Potential Functions, Scientia Iranica, Vol. 18, pp. 1138-1147, 2011.

[48] M. Kothari, I. Postlethwaite, D. Gu, Multi-UAV Path Planning in Obstacle Rich Environments Using Rapidly-exploring Random Trees, Proceedings of the 48th Conference on Decision and Control, pp. 3069-3074, 2009.

[49] B.D. Luders, S. Karaman, E. Frazzoli, J. P. How, Bounds on Tracking Error Using Closed-loop Rapidly-exploring Random Trees, American Control Conference, pp. 5406-5412, 2010.

[50] Z. Fang, Zh. Qidan, Path Optimization of Manipulator Based on the Improved Rapidly-exploring Random Tree Algorithm, Journal of Mechanical Engineering, pp. 123-132. 2012.

[51] M. Kothari1, I. Postlethwaite, D. Gu, A Suboptimal Path Planning Algorithm Using Rapidly-exploring Random Trees, Journal International Journal of Aerospace Innovations, Vol. 2, pp. 93-104, 2010.

[52] L. Qing, T. Xinhai, X. Sijiang, Z. Yingchun, Optimum Path Planning for Mobile Robots Based on a Hybrid Genetic Algorithm, Proceedings of the Sixth International Conference on Hybrid Intelligent Systems, pp. 53-58, 2006.

[53] L.A. Wilson, M. D. Moore, J. P. Picarazzi, S. D. Miquel, Parallel Genetic Algorithm for Search and Constrained Multi-objective Optimization, Proceedings of the Parallel and Distributed Processing Symposium, pp. 165, 2004.

[54] T. Sun, H. Pei, Y. Pan, C. Zhang, Robust Adaptive Neural Network Control for Environmental Boundary Tracking by Mobile Robots, International Journal of Robust and Nonlinear Control, in press, DOI: 10.1002/rnc.1816, 2012.

[55] Ch. Kai-Hui, M. R. Lee, Obstacle Avoidance in Mobile Robot Using Neural Network, International Conference on Consumer Electronics, Communications and Networks, pp. 5082-5085, 2011.

[56] S.M. Raguraman, D. Tamilselvi, N. Shivakumar, Mobile Robot Navigation Using Fuzzy Logic Controller, Control, Automation, Communication and Energy Conservation, pp. 1-5, 2009.

[57] U. Farooq, K.M. Hasan, G. Abbas, M.U. Asad, Comparative Analysis of Zero Order Sugeno and Mamdani Fuzzy Logic Controllers for Obstacle Avoidance Behavior in Mobile Robot Navigation,
International Conference and Workshop on Current Trends in Information Technology, pp. 113-119, 2011.

[58] K. Zhou, S. Ma, X. Zhu, L. Tang, X. Feng, Improved Ant Colony Algorithm based on cellular Automata for obstacle avoidance in robot soccer, International Conference on Computer Science and Information Technology, Vol.5, pp. 298-302, 2010.

[59] L. Zheng, L. Fei, X. Deng, Soccer Robot Based on Ant Colony Method of Obstacle Avoidance, International Conference on Consumer Electronics, Communications and Networks, pp. 4408-4412, 2011.

[60] M. Saska, M. Macas, L. Preucil, L. Lhotska, Robot Path Planning using Particle Swarm Optimization of Ferguson Splines, Proceedings of Conference on Emerging Technologies and Factory Automation, pp. 833-839, 2006.

[61] C. Xin, L. Yangmin, Smooth Path Planning of a Mobile Robot Using Stochastic Particle Swarm Optimization, Proceedings of International Conference on Mechatronics and Automation, pp. 1722-1727, 2006.

[62] N.L. Doh, N. Cho, K. Lee, J. Lee, W.K. Chung, S.R. Oh, A Systematic Representation of Edges in Topological Maps for Mobile Robots using Wavelet Transformation, Proceedings of International Conference on Robotics and Automation, pp. 2822-2827, 2005.

[63] E. Masehian, A.M.R. Naseri, A Tabu Search based Approach for Online Motion Planning, International Conference on Industrial Technology, pp. 2756 - 2761, 2006.

[64] Zh. Xin; Y. Maode, Y. Ju, A Tabu Search Based Flocking Algorithm of Motion Control for Multiple Mobile Robots, International Conference on Intelligent Computation Technology and Automation, pp. 48-52, 2012.

M. Nazemizadeh was born in Isfahan Iran. He received his B.Sc. in Mechanical Engineering from the Shahrekord University. He has also obtained his master's degrees in mechanical engineering at the Iran University of Science and Technology. He is now an education instructor in Islamic Azad Universtiy, and he is a Phd student in mechanical engineering at Amirkabir university of Iran, too.

$\mathrm{He}$ is interested in robotics researches and his works are mainly concentrated on obstacle avoidance and optimal path planning of manipulators and mobile robots. He has published about 15 papers on international and national journals and conferences, until now. 